\title{
Sentinel node investigation in breast cancer: detailed analysis of the yield from step sectioning and immunohistochemistry
}

\author{
H Torrenga, F D Rahusen, S Meijer, P J Borgstein, P J van Diest
}

\begin{abstract}
Aims-To evaluate in detail the extent to which step sectioning and immunohistochemical examination of sentinel lymph nodes (SNs) in patients with breast cancer reveal additional node positive patients, to arrive at a sensitive yet workable protocol for histopathological SN examination.

Methods-This study comprised 86 women with one or more positive $\mathrm{SN}$ after a successful $\mathrm{SN}$ procedure for clinical stage T1-T2 invasive breast cancer. SNs were lamellated into pieces of approximately $0.5 \mathrm{~cm}$ in size. One initial haematoxylin and eosin $(H \& E)$ stained central cross section was made for each block. When negative, four step ribbons were cut at intervals of $250 \mu \mathrm{m}$. One section from each ribbon was stained with $H \& E$, and one was used for immunohistochemistry (IHC).
\end{abstract}

Results-When taking the cumulative total of detected metastases at level 5 as $100 \%$, the percentage of $\mathrm{SN}$ positive patients increased from $80 \%, 83 \%, 85 \%$, $87 \%$ to $88 \%$ in the $H \& E$ sections through levels 1 to 5, and with IHC these values were $86 \%, 90 \%, 94 \%, 98 \%$, and $100 \%$. Three of nine patients in whom metastases were detected at levels 3-5 only had metastases in the subsequent axillary lymph node dissection.

Conclusions-Multiple level sectioning of SNs (five levels at $250 \mu \mathrm{m}$ intervals) and the use of IHC detects additional metastases up to the last level. Although more levels of sectioning might increase the yield even further, this protocol ensures a reasonable workload for the pathologist with an acceptable sensitivity when compared with the published literature. (f Clin Pathol 2001;54:550-552)

Keywords: breast cancer; sentinel node; immunohistochemistry; pathology

Sentinel node (SN) biopsy is rapidly gaining acceptance as a staging procedure in breast cancer. ${ }^{1-3}$ When the $\mathrm{SN}$ is identified successfully, the absence of tumour in this node predicts with a high degree of accuracy the absence of metastases in the remaining axillary lymph nodes. ${ }^{4-9}$

The reliability of the $\mathrm{SN}$ procedure as an accurate staging procedure is dependent on the ability to identify the true SN and the extent of histopathological examination of the SN. Both are instrumental in limiting the false negative rate of the $\mathrm{SN}$ procedure.

Multiple level sectioning has been shown to increase the detection rate of SN metastases in several studies. ${ }^{10-13}$ However, complete serial sectioning would result in an unacceptable workload for the pathologist. Therefore, a compromise must be found between workload and sensitivity by limiting the degree of step sectioning and immunohistochemistry (IHC) on multiple levels. Up to now, no consensus exists on the most (cost-)effective protocol. ${ }^{9}{ }^{14-17}$ Furthermore, no studies have yet reported on the precise yield of each additional level of step sectioning.

Our study was performed to evaluate in detail the yield of multiple levels and the use of IHC for detecting metastases in SNs, to arrive at a protocol for optimal SN investigation.

\section{Methods}

From a total of 250 consecutive women with clinical stage T1-T2 NOM0 invasive breast cancer who underwent a successful SN procedure from October 1994 to October 1999, we identified 86 patients in whom one or more $\mathrm{SNs}$ proved to be positive. The mean age of the patients was 56 years (range, 33-84), and pathological tumour size was on average $2 \mathrm{~cm}$ (range, 0.6-6).

We evaluated the extent of SN processing required before these patients were found to be positive. All patients who were initially negative by haematoxylin and eosin (H\&E) on the first $\mathrm{SN}$ section subsequently underwent standard multiple sectioning at $250 \mu \mathrm{m}$ intervals and staining with both $\mathrm{H} \& \mathrm{E}$ and IHC. We assessed at which additional level patients who were initially found to be negative were subsequently converted to SN positive.

SENTINEL LYMPH NODE BIOPSY

The day before surgery, $40 \mathrm{MBq}$ of $99 \mathrm{mTc}$ colloidal albumin was injected in two to four depots peritumorally. Lymphoscintigraphy was done to detect the presence, location, and number of focal accumulations.

Just before surgery, $0.5 \mathrm{ml}$ of $2.5 \%$ patent blue solution (Guerbet, Aulnay-sous Bois, France) was injected intracutaneously just around the areola. During surgery, axillary focal tracer accumulations were localised using a handheld $\gamma$ probe (c-track; Carewise, Morgan Hill, California, USA). All hot and blue nodes were removed as an SN. Furthermore, all radioactive nodes were biopsied until less than $10 \%$ of residual radioactivity, compared with the activity of the hottest $\mathrm{SN}$, remained in the axilla. 
Table 1 Cumulative number of patients with breast cancer sentinel node metastases found with each additional level (250 $\mu \mathrm{m}$ intervals) in a total of 86 patients

\begin{tabular}{lllll}
\hline & HEE & $\%$ & IHC & $\%$ \\
\hline Level 1 & 69 & 80 & 74 & 86 \\
Level 2 & 71 & 83 & 77 & 90 \\
Level 3 & 73 & 85 & 81 & 94 \\
Level 4 & 75 & 87 & 84 & 98 \\
Level 5 & 76 & 88 & 86 & 100 \\
\hline
\end{tabular}

H\&E, haematoxylin and eosin staining; IHC, CAM5.2 immunohistochemistry.

TISSUE PROCESSING

SNs smaller than $0.5 \mathrm{~cm}$ were processed intact, those between 0.5 and $1 \mathrm{~cm}$ were halved, and SNs larger than $1 \mathrm{~cm}$ were lamellated into pieces of approximately $0.5 \mathrm{~cm}$ in size. In most cases, intraoperative fresh frozen section analysis of all individual pieces was performed. ${ }^{19}$ After frozen section analysis, the SN was fixed in neutral buffered formaldehyde and embedded completely. One initial $4 \mu \mathrm{m}$ thick H\&E stained section was made for each block. When negative, an additional section was done at the first level for IHC and four step ribbons were cut at an interval of $250 \mu \mathrm{m}$. From these ribbons one section was stained with $\mathrm{H} \& \mathrm{E}$, and one was used for IHC with the CAM5.2 antibody (Becton Dickinson, San Jose, California, USA). All slides were examined by the same pathologist (PJvD), taking the frozen section and its control paraffin wax embedded section as level 1 .

All SNs containing any cell consistent with an epithelial morphology (and immunophenotye) were considered metastase positive.

\section{Results}

Of the 86 patients eventually found to have positive sentinel nodes, 69 patients were found to be positive on the first $\mathrm{SN}$ section stained with $\mathrm{H} \& \mathrm{E}$. Additional step sectioning and IHC were required in the remaining $20 \%$ of patients.

Interestingly, all 15 patients with more than one positive sentinel node were found to have SN metastases on the first H\&E stained section. The percentage of SN positive patients increased from $80 \%, 83 \%, 85 \%, 87 \%$ to $88 \%$ in the H\&E sections through levels 1 to 5 , and with IHC these figures were $86 \%, 90 \%, 94 \%$, $98 \%$ and $100 \%$. At the first level, IHC revealed five additional SN positive patients and the additional levels 2 to 5 revealed metastases in three, three, four, and two more patients, respectively (table 1 ). Of the nine patients with SN metastases only detected at levels 3-5, three had further metastases in the subsequent axillary lymph node dissection.

Looking at all harvested nodes in these 86 SN positive patients, a total of 103 tumour positive SNs were found. When taking the cumulative total of detected metastases at level 5 as $100 \%$, the percentage of positive SNs increased from $81 \%, 83 \%, 85 \%, 86 \%$ to $87 \%$ in the H\&E sections through levels 1 to 5. With IHC, the increase was from $86 \%, 90 \%, 94 \%$, $98 \%$ to $100 \%$. The first level failed to detect metastases in 14 SNs. Additional levels 2 to 5 yielded metastases in four, four, four, and two more positive SNs, respectively (table 2).
Table 2 Cumulative number of breast cancer sentinel node metastases found with each additional level (250 $\mathrm{mm}$ intervals) in 103 sentinel nodes

\begin{tabular}{lllll}
\hline & HEE & $\%$ & IHC & $\%$ \\
\hline Level 1 & 84 & 81 & 89 & 86 \\
Level 2 & 86 & 83 & 93 & 90 \\
Level 3 & 88 & 85 & 97 & 94 \\
Level 4 & 89 & 86 & 101 & 98 \\
Level 5 & 90 & 87 & 103 & 100 \\
\hline
\end{tabular}

H\&E, haematoxylin and eosin staining; IHC, CAM5.2 immunohistochemistry.

\section{Discussion}

Because validation studies have shown high accuracy rates for the $\mathrm{SN}$ procedure, ${ }^{4-9}$ it can now be used as a staging procedure without the need for complete axillary lymph node dissection in patients with a negative $\mathrm{SN}$. $^{1}$

Provided that the surgeon has performed a reliable SN biopsy procedure, it is up to the pathologist to determine whether axillary metastases are present or not.

In the days of routine axillary dissection, it was shown that a large proportion of patients are converted from node negative to node positive with more elaborate histological examination of axillary nodes. ${ }^{162021}$ This has important consequences for the clinical management of these patients because, in general, lymph node positive patients will receive adjuvant treatment. For these reasons, the histopathological examination of $\mathrm{SNs}$ tends to be even more elaborate, with the additional argument that it can be done so with an acceptable workload for the pathologist because only a few nodes need to be examined this way.

Our study clearly shows that more patients are converted to node positive with each additional step of sectioning (at $250 \mu \mathrm{m}$ intervals) and that with each additional step IHC has a higher sensitivity than H\&E. The clinical relevance of finding these metastases is underlined by the fact that several patients with $\mathrm{SN}$ metastases, only visible at level 3-5, had second echelon metastases.

However, even with this intensive protocol not all nodal metastases will be detected. Such a $100 \%$ sensitivity can theoretically only be reached with complete serial sectioning of the $\mathrm{SN}$ at $12 \mu \mathrm{m}$ intervals. ${ }^{22}$

However, the question arises whether one really needs to find all metastatic cells. Therefore, one needs to arrive at a method that has a high sensitivity with an acceptable workload for the pathologist.

In a few studies complete serial sectioning of the $\mathrm{SN}$ has been done. For instance, Cserni ${ }^{11}$ serially sectioned the $\mathrm{SN}$ up to extinction with 3-5 $\mu \mathrm{m}$ thick slices and examined every 10 th to 20th level. In the final analysis, 15 of the 21 patients with metastases limited to the $\mathrm{SN}$ were positive on the initial central cross section. Dowlatshahi et al performed complete serial sectioning at $250 \mu \mathrm{m}$ intervals and found that only six of $30 \mathrm{SN}$ positive patients were positive on the initial section examined by $\mathrm{H} \& \mathrm{E}$ and IHC. ${ }^{23}$ This difference is remarkable, also in view of the fact that Cserni's intervals were smaller and therefore it would be expected that a greater number of additional metastases 
would be found. The greater yield on the initial section in Cserni's study could be ascribed to the fact that they tried to cut the central cross section guided by the blue lymphatic vessel. ${ }^{24}$ In addition, the average size of the metastases may have been greater in patients in Cserni's study $(68 \% \mathrm{~T} 2$ tumours versus $25 \%$ in the study by Dowlatshahi et al).

Nevertheless, the fact remains that the use of IHC and additional step sectioning improves the detection rate of metastatic deposits. The question for practical purposes then remains: how many additional levels (and at what interval) need to be examined?

Turner et al examined 10 levels at $40 \mu \mathrm{m}$ intervals in $42 \mathrm{SN}$ positive patients in whom the initial $\mathrm{H} \& \mathrm{E}$ section was negative..$^{10}$ In these patients, with the use of IHC the first two levels of SN examination found additional metastases in all but one patient. They therefore concluded that the additional eight levels of examination did not significantly contribute to the detection of additional metastases. However, with 10 levels at $40 \mu \mathrm{m}$ intervals only $400 \mu \mathrm{m}$ of the entire $\mathrm{SN}$ is examined, which might not be sufficient for the detection of all metastases. Rather than taking many sections at small intervals, it may be more efficient to take fewer sections at larger step intervals.

Our present study shows in detail the yield of step sectioning and IHC at five levels with an interval of $250 \mu \mathrm{m}$. Because we routinely perform frozen section analysis, which leads to some loss of material, this ensures sampling through the larger part of the SN. In practice, this has proved to be an acceptable workload. Clearly, the yield increased with additional levels. The first level failed to detect metastases in 14 SNs $(14 \%$ of the total number of metastases found). Additional levels 2 to 5 yielded metastases in four, four, four, and two more SNs, respectively. Thus, additional levels clearly reveal more metastases and even the fourth and fifth level together reveal $6 \%$ additional metastases. Not surprisingly, the yield with IHC was higher than with $\mathrm{H} \& \mathrm{E}$ only. IHC facilitates the detection of single metastatic cells, and speeds up the screening of the sections dramatically. Therefore, some investigators omit H\&E staining when IHC is performed. However, we prefer to make $H \& E$ control sections because they are helpful in the detection of artefacts and benign inclusions.

In conclusion, step sectioning of SNs with ICH is very useful for finding the smallest metastases in SNs of breast cancer that may be clinically relevant. We therefore propose step sectioning and ICH at four additional levels, separated by $250 \mu \mathrm{m}$ intervals, when the original H\&E section is tumour negative. Despite the fact that our protocol might still miss some metastases we believe this protocol ensures a reasonable workload for the pathologist with an acceptable sensitivity.

1 Rahusen FD, Pijpers R, Diest PJ van, et al. The implementation of the SN biopsy as a routine procedure in breast cancer patients. Surgery 2000;128:6-12.

2 Giuliano AE. See one, do twenty-five, teach one: the implementation of sentinel node dissection in breast cancer. Ann mentation of sentinel node

3 Cody HS, 3rd, Borgen PI. State-of-the-art approaches to sentinel node biopsy for breast cancer: study design, patient selection, technique, and quality control at Memorial Sloan-Kettering Cancer Center. Surg Oncol 1999;2:85-91.

4 Borgstein PJ, Pijpers R, Comans EFI, et al. Sentinel lymph node biopsy in breast cancer: guidelines and pitfalls of lymphoscintigraphy and gamma probe detection. $7 \mathrm{Am}$ Coll Surg 1998;186:275-83.

5 Krag D, Weaver D, Ashikaga T, et al. The sentinel node in breast cancer-a multicenter validation study. $N$ Engl $\mathcal{F}$ Med 1998;339:941-6.

6 Giuliano AE, Kirgan DM, Guenther JM, et al. Lymphatic mapping and sentinel lymphadenectomy for breast cancer. Ann Surg 1994;3:391-8.

7 Veronesi U, Paganelli G, Galimberti V, et al. Sentinel node biopsy to avoid axillary dissection in breast cancer with clinically negative lymph node. Lancet 1997;349:1864-7.

8 Cox CE, Bass SS, McCann CR, et al. Lymphatic mapping ox CE, Bass SS, McCann CR, et al. Lymphatic mapping
and sentinel node biopsy in patients with breast cancer. Annu Rev Med 2000;51:525-42.

Annu Rev Med 2000;51:525-42.
9 Turner RR, Ollila DW, Krasne DL, et al. Histopathologic Turner RR, Ollila DW, Krasne DL, et al. Histopathologic
validation of the sentinel lymph node hypothesis for breast carcinoma. Ann Surg 1997;226:271-8.

10 Turner RR, Ollila DW, Stern S, et al. Optimal histopathologic examination of the sentinel lymph node for breast carcinoma staging. Am f Surg Pathol 1999;23:263-7.

11 Cserni G. Metastases in axillary sentinel lymph nodes in breast cancer as detected by intensive histopathological workup. 7 Clin Pathol 1999;52:922-3.

12 Weaver DL, Krag DN, Ashikaga T, et al. Pathologic analysis of sentinel and nonsentinel lymph nodes in breast

;8:1099-107.

Jannink I, Fan M, Nagy S, et al. Serial sectioning of sentinel nodes in patients with

14 Veronesi U, Paganelli G, Viale G, et al. Sentinel lymph node biopsy and axillary dissection in breast cancer: results in a biopsy and axillary dissection in breast cancer:

15 Snider H, Dowlatshahi K, Fan M, et al. Sentinel node biopsy in staging of breast cancer. Am F Surg 1999;176:305-10.

16 Diest PJ van, Peterse HL, Borgstein PJ, et al. Pathologic investigation of sentinel lymph nodes. Eur $\mathcal{F}$ Nucl Med 1999;26:S43-9.

17 Torrenga H, Rahusen FD, Borgstein PJ, et al. Immunohistochemical detection of lymph-node metastases. Lancet 2000;335:144

18 Withdrawn

19 Diest PJ van, Torrenga H, Borgstein PJ, et al. Reliability of intra-operative frozen section and imprint cytological investigation of sentinel lymph nodes in breast cancer. Histopathology 1999;35:14-18.

20 Cote RJ, Peterson HF, Chaiwun B, et al. Role of immunohistochemical detection of lymph node metastases in management of breast cancer. Lancet 1999;354:896900 .

21 Wilkinson EJ, Hausse LL, Hoffman RG, et al. Occult axillary lymph node metastases in invasive breast carcinoma: characteristics of the primary tumour and significance of the metastases. Pathol Anпu 1982;2:67-91. 2 Diest PJ van. Histopathologic workup of the sentinel nodes: how much is enough? F Clin Pathol 1999;52:871-3.

23 Dowlatshahi K, Fan M, Bloom KJ, et al. Occult metastases on the sentinel lymph nodes of patients with early stage breast carcinoma: a preliminary study. Cancer 1999;86: $990-6$.

24 Cserni G. Mapping metastases in sentinel lymph nodes of breast cancer. Am f Clin Pathol 2000;113:351-4. 